\title{
Erector spinae plane block (ESP) for postoperative analgesia for mastectomy total radical: case report
}

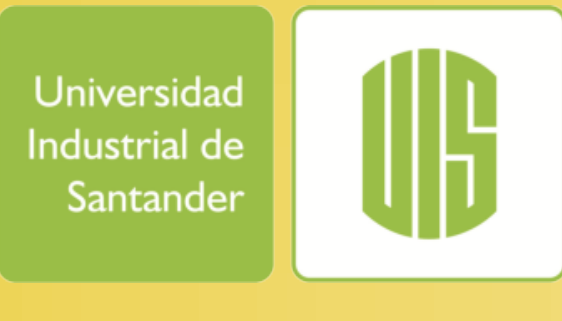

Dra Viviana P. Rueda Rojas, Elba Orozco, Rafael Serrano. Universidad Industrial de Santander, Anestesiologìa, Bucaramanga, Colombia.

\section{Background and Aims}

Postoperative pain control in breast cancer surgery has become one of the most important goals for anesthetists. Ultrasound-guided erector spinaeplane (ESP) block is an innovative, easy, safe.

\section{Methods}

To describe 50-year-old female patient, , a weight of $62 \mathrm{~kg}$, with an 8-year history of a mass in the right breast which has undergone rapid progressive growth, involving the entire breast, over the past 7 months, diagnosed as a phyllodes tumor. Simple right mastectomy with axillary lymph node dissection and pectoralis major fascia resection were performed under general anesthesia. Ultrasound-guided erector spinae plane block was performed for postoperative analgesia, with excellent response up to 18 hours following the procedure.

\section{Results}

The patient was placed on left lateral decubitus and, following asepsia and anti-sepsis, T6 to T7 and right paravertebral ultrasound was performed using a highfrequency linear probe, to identify the anatomy (Figure 2) Bupivacaine with $0.25 \%$ epinephrine and $0.5 \%$ lidocaine was administered (total volume $20 \mathrm{~mL}$ ), achieving satisfactory erector spinae hydrodissection. Assessment 4hours later found an area of anesthesia comprised by the right anterior hemithorax and axillary region and anesthesia from T4 to T12 in the posterior region (Figure 3) Pain assessment was performed over a 30 -hour period using the analog visual scale.

Based on our findings, ESP block may be recommendedas an option or adjunct for pain management.
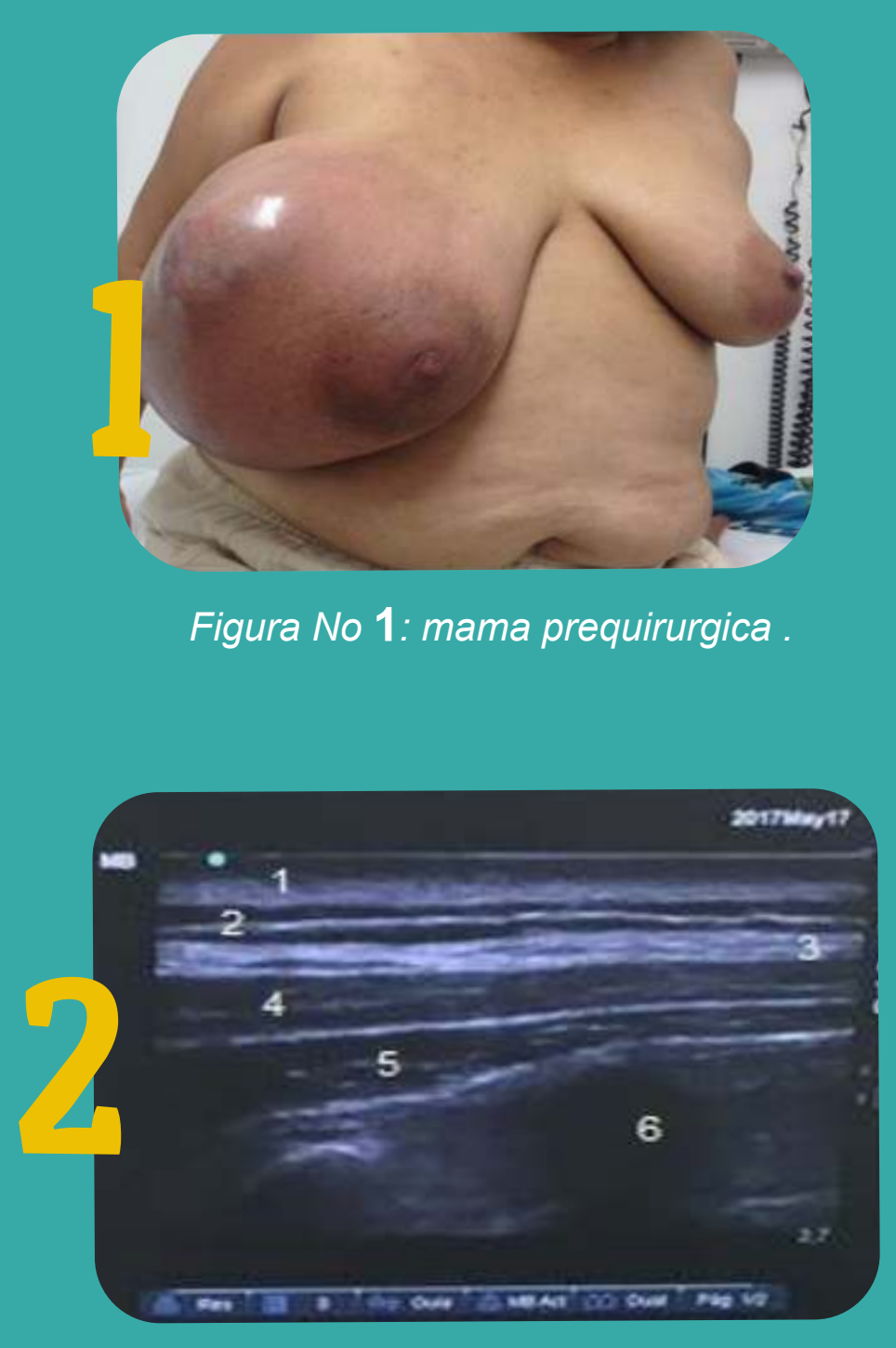

Figure 2: Ultrasound image of ESP block. $E S P=$ erector spinae plane: Identifies from the outside to the inside: 1.Skin, 2.subcutaneous cellular tissue, 3.Trapezius muscle, 4. rhomboid major muscle, 5.Erector Spinae muscle, 6.transverse process. A 50- $\mathrm{mm}$ echosensitive needle was placed through an in-plane cephalocudal approach up to the erector spinae muscle.

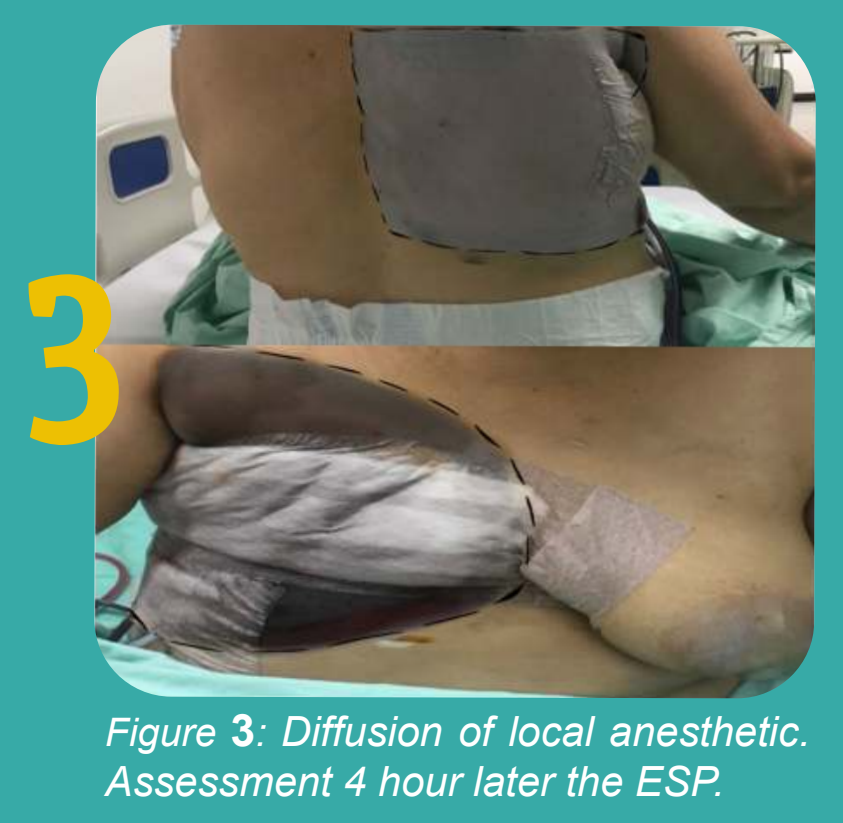

\begin{tabular}{|c|c|c|c|c|c|c|c|}
\hline \multirow{3}{*}{ Case } & \multirow{3}{*}{$\begin{array}{c}\text { Sensory block } \\
\text { ares }\end{array} \mid$} & \multicolumn{5}{|c|}{$\begin{array}{c}\text { Pain intensity } \\
\text { assessment VAS }\end{array}$} & \multirow{3}{*}{$\begin{array}{c}\text { Use of morphine } \\
\text { PCA }\end{array}$} \\
\hline & & \multicolumn{5}{|c|}{ Hours } & \\
\hline & & 4 & 12 & 18 & 24 & 30 & \\
\hline 1 & T6-T12 & 0 & 0 & 1 & 1 & 2 & $\begin{array}{l}1 \mathrm{mg} \text { in } \\
30 \text { hours }\end{array}$ \\
\hline
\end{tabular}

\section{Conclusions}

ESP block is a safe, innovative strategy that is easy to perform and ensures good postoperative analgesia in radical mastectomy, reducing opioid requirements. It offers good pain management, contributing to faster patient recovery.

\section{Bibliography}

1. Forero M, Rajarathinam M, Adhikary S, Chin K J. Continuous erector spinae plane block for rescue analgesia in thoracotomy after epidural failure: a case report. AA Case Rep 2017; 8(10): 254-6. 2.Forero M, Adhikary SD, López H, Tsui C, Chin KJ. The erector Spinae plano block: a novel analgesic technique in thoracic neuropatic pain. Reg Anesth Pain Med 2016;41(5): 621 - 7 3.Krediet, AC., Moayeri N, van Geffen GJ, Bruhn J, Renes S, Bigeleisen PE, Groen GJ. Different Approaches to ultrasound-guided thoracic paravertebral Block: an ilustrated review. Anesthesiology 2015; 123(2): $459-474$. 